\title{
Sarcoidosis del adulto de inicio en la infancia: a propósito de un caso
}

Pediatric-onset adult type sarcoidosis: A case report

Dra. Yasemin Ozsurekci ${ }^{a}$ Dr. Ali B. Cengiz ${ }^{a}$ Dr. Ali Duzovab, Dr. Erdal Sagc , Dra. Sibel Kadayıfcilar ${ }^{d}$, Dra. Deniz Dogru Ersoze, Dra. Zuhal Akcorenf, Dra. Aysel Yuces, Dra. Betul Tavilh, Dra. Deniz Ayvazi,

Dra. Canan Akyuz' y Dra. Fehime Kara Eroglu

\section{RESUMEN}

La sarcoidosis, un trastorno multiorgánico de etiología desconocida que afecta varios órganos, es poco frecuente en los niños. Se desconocen la incidencia y la prevalencia reales de la sarcoidosis infantil. Al igual que en los adultos, muchos niños con sarcoidosis tal vez no presentan síntomas y la enfermedad cursa sin diagnosticarse. Es fundamental realizar una evaluación completa y sistemática del paciente para establecer el diagnóstico de sarcoidosis en los niños. Se describe el caso de una niña de 12 años con uveítis y hepatoesplenomegalia de dos años de evolución. Mediante una tomografía computarizada del tórax, se hallaron nódulos pulmonares periféricos dispersos y linfadenopatía hiliar bilateral. La aspiración de médula ósea y la biopsia de hígado no fueron diagnósticas.

Labiopsia de pulmón mostró granulomas de células epitelioides no necrosantes. A la paciente se le diagnosticó sarcoidosis en virtud del hallazgo de inflamación granulomatosa y de la exclusión de entidades confusoras.

Palabrasclave: sarcoidosis pulmonar; niños; uveítis; hepatomegalia; esplenomegalia.

http:/ /dx.doi.org/10.5546/aap.2015.e336

\section{INTRODUCCIÓN}

La sarcoidosis infantil es un trastorno granulomatoso multiorgánico idiopático y poco

a. Departamento de Enfermedades Infecciosas Pediátricas.

b. División de Reumatología Pediátrica.

c. Departamento de Pediatría.

d. Departamento de Oftalmología.

e. División de Neumología Pediátrica.

f. División de Patología Pediátrica.

g. División de Gastroenterología Pediátrica.

h. División de Hematología Pediátrica.

i. División de Inmunología Pediátrica.

j. División de Oncología Pediátrica.

Facultad de Medicina de la Universidad Hacettepe,

Ankara, Turquía.

Correspondencia:

Dra. Yasemin Ozsurekci: yas.oguz99@yahoo.com

Financiamiento: Ninguno.

Conflicto de intereses: Ninguno que declarar.

Recibido: 10-4-2015

Aceptado: 10-6-2015 frecuente, habitualmente caracterizado por síntomas generales inespecíficos, manifestaciones pulmonares, linfadenopatía, y compromiso cutáneo y ocular. ${ }^{1,2}$ Se ha descripto la presentación clínica de la sarcoidosis en series pequeñas de niños de diferentes etnias. La evolución y el pronóstico de la sarcoidosis en niños difieren de lo que sucede en los adultos, y tal vez se correlacionen con la forma en que se manifiesta y el alcance de la enfermedad. Se desconocen la incidencia y la prevalencia reales de la sarcoidosis infantil debido a su infrecuencia y a la poca cantidad de casos informados durante la niñez. ${ }^{2}$ Los lactantes y niños menores de cinco años suelen presentar una tríada de síntomas cutáneos, oculares y en las articulaciones sin la enfermedad pulmonar característica. Sin embargo, en los niños mayores de cinco años, se ven afectados los pulmones, los ganglios linfáticos y los ojos con más frecuencia, como en los adultos. ${ }^{1}$

En 2001, se halló una mutación en el gen NOD2/CARD15 (dominio de oligomerización unido a nucleótidos $2 /$ dominio de reclutamiento de caspasa activada 15) en los pacientes con antecedentes de artritis granulomatosa familiar. A partir de este hallazgo surgió una nueva perspectiva y fue posible comprender la complejidad y heterogeneidad de la sarcoidosis infantil. ${ }^{3}$ El síndrome de Blau y la sarcoidosis de inicio temprano son las formas familiares y esporádicas de la sarcoidosis infantil caracterizada por la asociación con mutaciones en el gen NOD2. ${ }^{4}$ Sin embargo, en muchos casos pediátricos en los que se diagnosticó sarcoidosis, no se halló una mutación en el gen NOD2 y, en general, presentan manifestaciones sistémicas y viscerales. Dentro de este grupo, existen dos entidades identificadas: la paniculitis con granulomatosis sistémica de aparición en la niñez y la sarcoidosis del adulto de inicio en la infancia. ${ }^{5}$

El diagnóstico temprano de la sarcoidosis en los niños es, a veces, difícil debido a la falta de conocimiento y familiarización con sus características clínicas. ${ }^{6}$ Informamos el caso 
de una niña de 12 años con diagnóstico de sarcoidosis del adulto de inicio en la infancia.

\section{CASO CLÍNICO}

En noviembre de 2012, se ingresó a una niña de 12 años en la Unidad de Enfermedades Infecciosas Pediátricas de la Facultad de Medicina de la Universidad Hacettepe; tenía febrícula recurrente, linfadenopatía periférica múltiple y distensión abdominal de 1,5 años de evolución. También informó tener malestar general e inapetencia. No mostró mejoría con el tratamiento empírico con diversos antibióticos, entre otros, betalactámicos. Sus antecedentes médicos incluían uveítis dos años antes, aunque sus antecedentes familiares no presentaban particularidades.

Según el examen físico, se trataba de una niña de aspecto adecuado y signos vitales normales. Presentaba linfadenopatía cervical y axilar, y hepatoesplenomegalia. En la ecografía abdominal se observó el aumento de la longitud del hígado $(13 \mathrm{~cm})$ y del bazo $(12 \mathrm{~cm})$. Los ganglios linfáticos eran, en general, duros, sin dolor a la palpación y se movían libremente. El examen oftalmológico mostró agudeza visual de 1,0 en ambos ojos. El examen con lámpara de hendidura del ojo derecho era normal; sin embargo, se observaron +1 células en la cámara anterior y precipitados queráticos, con sinequia posterior en el ojo izquierdo (Figura 1). La oftalmoscopía mostró envainamiento perivascular, especialmente marcado en el ojo izquierdo. La angiografía con fluoresceína de retina confirmó la presencia de vasculitis segmentaria focal en la periferia de ambos ojos (Figura 2). El resto del examen físico era normal. En los análisis de laboratorio iniciales se observó concentración de hemoglobina

Figura 1. El examen con lámpara de hendidura del ojo derecho era normal; sin embargo, se observaron +1 células en la cámara anterior y precipitados queráticos, con sinequia posterior en el ojo izquierdo

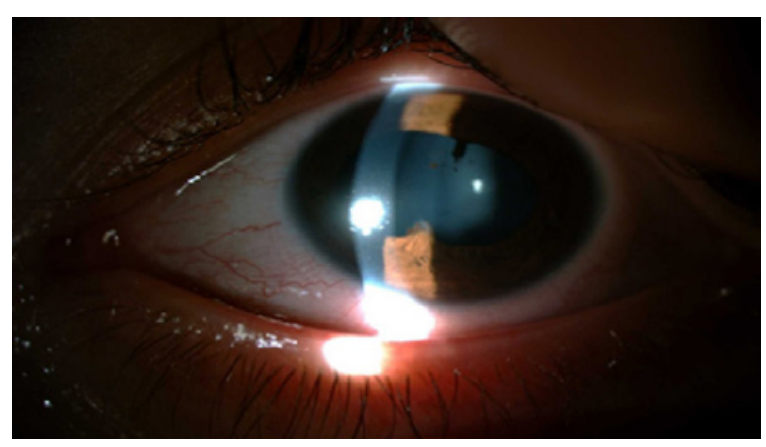

de 9,7 g/dl (rango: 11,7-15,5), recuento de trombocitos de $377000 / \mathrm{ml}$ (rango: 150 000388 000), recuento de leucocitos de $5800 / \mathrm{ml}$ (rango: 4100-11 200), proteína $C$ reactiva (PCR) de $0,26 \mathrm{mg} / \mathrm{dl}$ (rango: 0-0,8), y velocidad de sedimentación globular de $51 \mathrm{~mm} / \mathrm{h}$ (normal $<20)$. No presentaba eosinofilia, y el frotis periférico era normal. El análisis bioquímico de sangre de las pruebas funcionales renales y hepáticas y el análisis de orina eran normales. El resultado de la prueba de la tuberculina fue negativo.

Las concentraciones séricas de $\operatorname{Ig} \mathrm{A}, \operatorname{IgG}$ e IgM eran 700 mg/dl (rango: 82-453), $1870 \mathrm{mg} / \mathrm{dl}$ (rango: 751-1560), y 647 mg/dl (rango: 46-304), respectivamente. Los análisis inmunológicos primarios, entre otros, análisis de subconjuntos de linfocitos y el examen de nitroazul de tetrazolio (NAT), eran normales, al igual que las inmunoglobulinas. La concentración de enzima convertidora de la angiotensina (ECA) era $75 \mathrm{U} / \mathrm{L}$ (rango: 7-50). Los resultados de autoanticuerpos, incluidos el anticuerpo antinuclear, anticuerpo anticitoplasma de neutrófilos y anticuerpo anticitoplasma de neutrófilos con patrón perinuclear, fueron negativos. La concentración sérica de calcio era de 9,3 mg/dl. En la ecografía cervical y abdominal se observó aumento de tamaño de las glándulas submandibulares y cervicales, con un diámetro máximo de $15 \mathrm{~mm}$, y hepatoesplenomegalia. Las pruebas de función pulmonar y la pletismografía pulmonar eran normales.

Se realizaron dos biopsias de médula ósea para descartar la presencia de tumores malignos y ciertas enfermedades infecciosas asociadas con el compromiso del sistema reticuloendotelial, como la leishmaniosis visceral. El análisis de las biopsias de médula ósea fue normal. En una

FIGURA 2. La oftalmoscopía mostró envainamiento perivascular, especialmente marcado en el ojo izquierdo. La angiografía con fluoresceína de retina confirmó la presencia de vasculitis segmentaria focal en la periferia de ambos ojos (a: ojo derecho, b: ojo izquierdo)

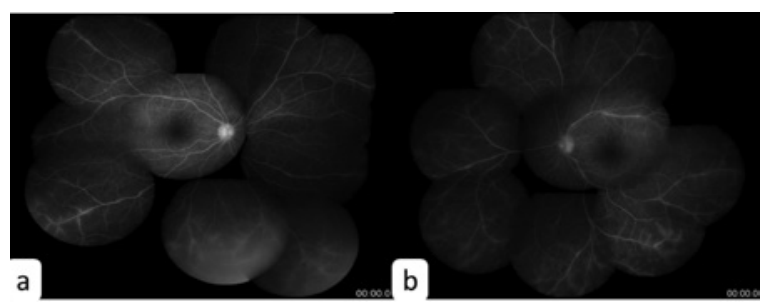


radiografía de tórax se observó adenopatía hiliar bilateral con compromiso del parénquima en los lóbulos inferiores (Figura 3 A). La tomografía computarizada (TC) del tórax mostró adenopatía hiliar bilateral (Figura 3 B1) y nódulos pulmonares periféricos bilaterales dispersos (Figura 3 B2).

En el examen físico, se observó que la linfadenopatía periférica se resolvió en unos días; por lo tanto, se realizó una biopsia de hígado para confirmar el diagnóstico. Sin embargo, en esta biopsia solamente se observó inflamación crónica sin formación de granulomas. Posteriormente, se realizó una biopsia de pulmón a cielo abierto, donde se observaron granulomas múltiples sin necrosis, con células gigantes multinucleadas rodeadas de linfocitos, compatible con sarcoidosis (Figura 4). Se realizaron tinciones especiales, para bacilos acidorresistentes y hongos, que fueron negativas, y también fue negativo el cultivo del tejido biopsiado para detectar bacilos aerobios. No se realizó el análisis de mutación del gen NOD2. A la paciente se le diagnosticó sarcoidosis y se inició la administración de prednisolona (1 mg/kg/día, por vía oral). Los hallazgos sistémicos y oculares de la paciente habían mejorado al final de un ciclo de corticoesteroides de tres meses. Se disminuyó progresivamente la dosis de corticoesteroides, y no se observaron complicaciones en el seguimiento durante seis meses.

\section{DISCUSIÓN}

La sarcoidosis es menos frecuente en los niños que en los adultos. En un estudio realizado en Dinamarca, de 15 años de duración, la incidencia

FiguRA 3. En una radiografía de tórax se observó adenopatía hiliar bilateral con compromiso del parénquima en los lóbulos inferiores (A). La tomografía computarizada de tórax con contraste mostró adenopatía hiliar bilateral (B1) y nódulos pulmonares periféricos dispersos (B2)

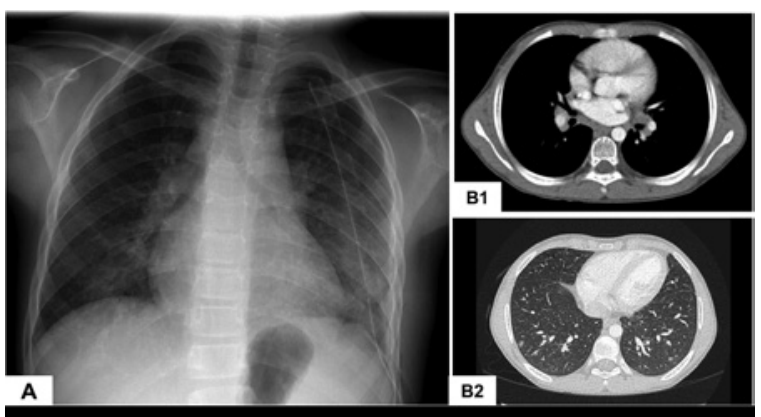

de sarcoidosis fue 0,06 casos cada 100000 niños menores de cuatro años de edad, con un aumento progresivo con la edad a 1,02 casos cada 100000 niños de 14 a 15 años de edad. ${ }^{7}$ Dado que la sarcoidosis es una enfermedad multiorgánica que afecta la mayoría de los órganos, la presentación clínica varía considerablemente. ${ }^{2}$

Si bien pueden producirse lesiones en cualquier tejido $u$ órgano, el pulmón es el órgano afectado más frecuentemente en la sarcoidosis. ${ }^{2}$ Nuestra paciente presentó, en primer lugar, afectación ocular, seguida de episodios recurrentes de fiebre y visceromegalia.

La afectación ocular tal vez sea la manifestación inicial de la sarcoidosis y puede derivar en el deterioro visual grave. ${ }^{7}$ Los niños menores de cinco años presentan una tríada clínica compuesta de artritis, lesiones cutáneas y uveítis. En el grupo de 8 a 15 años de edad, la evolución clínica es más similar a la de los adultos. ${ }^{8}$ Entre las manifestaciones oculares más frecuentes se incluyen la uveítis, característica temprana de la sarcoidosis, y los nódulos conjuntivales. Más del $80 \%$ de los casos de uveítis se manifestaron dentro del año posterior a la aparición de las enfermedades sistémicas. ${ }^{8}$ El tipo de uveítis asociada con la sarcoidosis es la uveítis anterior. La uveítis anterior con compromiso del segmento posterior y la enfermedad sistémica son factores pronósticos de una visión deficiente. ${ }^{9}$

FIGURA 4. Biopsia de pulmón que muestra inflamación granulomatosa sin necrosis con células gigantes multinucleadas (flecha gruesa), histiocitos epitelioides (punta de flecha) y linfocitos (flecha delgada), (tinción hematoxilina-eosina)

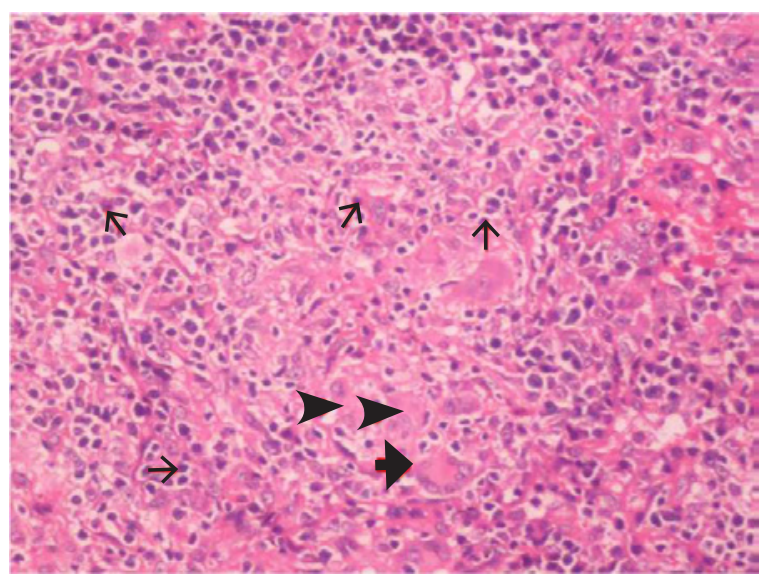


Nuestra paciente tenía uveítis anterior con compromiso del segmento posterior en ambos ojos. La angiografía con fluoresceína confirmó actividad en el segmento posterior, vasculitis segmentaria periférica y envainamiento perivascular.

En general, el síndrome de Blau o la sarcoidosis de inicio temprano (sarcoidosis asociada con mutación en el gen NOD2) se presentan con poliartritis, dermatitis y uveítis.

Las características principales de la sarcoidosis sin mutación en el gen NOD2, como la sarcoidosis del adulto de inicio en la infancia, son sistémicas y afectan los ganglios linfáticos y los pulmones. Asimismo, la incidencia tiende a aumentar durante los primeros años de la adolescencia. ${ }^{3-5}$ Si bien no fue posible realizar el análisis del gen NOD2, en nuestra paciente es probable que se tratara de sarcoidosis del adulto de inicio en la infancia, sin mutación en el gen NOD2 de acuerdo con el tipo de presentación clínica.

Si bien no hay un único análisis de laboratorio que permita diagnosticar la sarcoidosis, existen varios resultados complementarios, entre otros, aumento de los reactantes en la fase aguda, hipercalcemia, anemia, leucopenia y eosinofilia., ${ }^{2,10}$ Puede producirse un aumento significativo de la enzima convertidora de la angiotensina (ECA), que es útil como marcador de la actividad de la enfermedad, aunque esta prueba no es específica para la sarcoidosis. Los valores fisiológicos de la ECA varían según la edad, y los niños suelen tener un rango normal de concentraciones séricas más elevado. Por lo tanto, la concentración de ECA sigue siendo poco clara para el diagnóstico y tratamiento de la sarcoidosis. ${ }^{11}$

La concentración de ECA de nuestra paciente estaba cercana al límite superior del rango normal y no sirvió como marcador complementario del diagnóstico.

El pulmón es el órgano más frecuentemente afectado en la sarcoidosis. A menudo, el examen físico es aparentemente normal, y la linfadenopatía hiliar bilateral con o sin compromiso del parénquima es el hallazgo radiográfico más frecuente. ${ }^{12}$ Además de los hallazgos de la radiografía de tórax, es posible que la TC de tórax revele enfermedad del parénquima. El compromiso del parénquima suele presentar un patrón intersticial, aunque también se han descripto patrones nodulares, alveolares y fibróticos. ${ }^{13}$ En nuestra paciente, no se observaron síntomas pulmonares y el examen físico era aparentemente normal. Los hallazgos radiológicos eran compatibles con sarcoidosis, cuyo diagnóstico se confirmó mediante biopsia de pulmón. Una muestra de la biopsia confirmó el diagnóstico de sarcoidosis al mostrar granulomas de células epitelioides no caseificantes y al descartar otras causas conocidas de inflamación granulomatosa, como infecciones por hongos, micobacterias y parásitos, y entidades insignificantes, como la granulomatosis de Wegener. ${ }^{14}$

Asimismo, la sarcoidosis hepatoesplénica suele manifestarse más frecuentemente como visceromegalia, como en el caso de nuestra paciente. Entre los pacientes con sarcoidosis sistémica, del $24 \%$ al $94 \%$ tienen sarcoidosis hepática documentada mediante biopsia. En la sarcoidosis sistémica, el bazo suele verse más frecuentemente afectado que el hígado.

El compromiso esplénico se confirma en hasta el $60 \%$ de los pacientes mediante biopsia. ${ }^{15}$ Si bien se consideró el hígado para una biopsia inicial por ser un órgano de fácil acceso, no fue posible confirmar el diagnóstico de sarcoidosis con los hallazgos de la biopsia de hígado.

La adenopatía periférica fue frecuente ( $40 \%$ de 48 niños) en el estudio danés; $;^{10}$ y dicha localización ayudó con el diagnóstico en 15 de ellos. No obstante, en nuestro caso, la linfadenopatía periférica se resolvió durante la evaluación de la paciente, y fue necesario realizar una biopsia de hígado y otra de pulmón para lograr el diagnóstico definitivo.

En un niño con inflamación granulomatosa, es necesario descartar infecciones crónicas, incluidas las infecciones por micobacterias y hongos, mediante pruebas de tinción y cultivos razonables, para lograr el diagnóstico de sarcoidosis. Los pacientes con distintos trastornos de inmunodeficiencia primaria pueden presentar inflamación granulomatosa sin una causa infecciosa identificable, y se los debe excluir mediante evaluación de la función de los neutrófilos y análisis de los subconjuntos de linfocitos circulantes y las concentraciones séricas de inmunoglobulina. ${ }^{5,14}$ En el caso presentado, no hallamos una causa infecciosa al evaluar los cultivos y las tinciones de tejido. Además, en todos los análisis inmunológicos primarios de nuestra paciente, los subconjuntos de linfocitos e inmunoglobulinas estaban dentro de los límites normales. Por lo tanto, descartamos las entidades confusoras con el diagnóstico de sarcoidosis.

Hasta dos tercios de los pacientes no tratados tienen remisión espontánea en los dos primeros años después de la aparición de los síntomas. Se indican corticoesteroides para el tratamiento de 
las enfermedades multisistémicas o cuando un órgano específico está gravemente afectado, por ejemplo, los pulmones o los ojos, y para la sarcoidosis neurológica o cardíaca. Se han utilizado metotrexato y otros inmunosupresores, además de citotóxicos y otras terapias biológicas más recientes, como los agentes ahorradores de corticoesteroides, para reducir o prevenir los efectos secundarios relacionados con el tratamiento prolongado con corticoesteroides. ${ }^{2,10}$ En nuestra paciente, el tratamiento corticoesteroideo resultó en una buena evolución clínica.

La variada presentación clínica de la sarcoidosis, especialmente en relación con la edad, suele representar un desafío diagnóstico para los médicos. En consecuencia, el diagnóstico tardó dos años debido a la naturaleza de remisión y recidiva de nuestra paciente. La evaluación clínica y de laboratorio inmediata junto con estudios de diagnóstico por imágenes, seguidos de hallazgos en los tejidos, son fundamentales para lograr tanto el diagnóstico como el tratamiento tempranos de la sarcoidosis.

\section{REFERENCIAS}

1. Fauroux B,Clément A. Paediatric sarcoidosis. Paediatr Resir Rev 2005;6(2):128-33.

2. Shetty AK, Gedalia A. Childhood sarcoidosis: a rare but fascinating disorder. Pediatr Rheumatol Online J 2008;6: 16.

3. Miceli-Richard C, Lesage S, Rybojad M, Prieur AM, et al. CARD15 mutations in blau syndrome. Nat Genet 2001;29(1):19-20.

4. Rosé CD, Doyle TM, Mcllvain-Simpson G, Coffman JE, et al. Blau syndrome mutation of CARD15/NOD2 in sporadic early onset granulomatous arthritis. J Rheumatol 2005;32(2):373-5.

5. Rose CD, Wauters CH. Pediatric Sarcoidosis. In Cassidy JT, Petty RE, Laxer RM, Lindsley CB. Textbook of Pediatric Rheumatology. 6th ed. Canada:Saunders; 2011.Págs.54451.

6. Sharma SK, Soneja M, Sharma A, Sharma MC, Hari S. Rare manifestations of sarcoidosis in modern era of new diagnostic tools. Indian J Med Res 2012;135(5): 621-9.

7. Rothova A. Ocular involvement in sarcoidosis. $\mathrm{Br} \mathrm{J}$ Ophthalmol 2000;84(1):110-6.

8. Hoover DL, Khan JA, Giangiacomo J. Pediatric ocular sarcoidosis. Surv Ophthalmol 1986;30(4):215-28.

9. UmurKA, Tayfun B, Oguzhan O. Different ophthalmologic manifestations of sarcoidosis. Curr Opin Ophthalmol 2012;23(6):477-84.

10. Hoffmann AL, Milman N, Byg KE. Childhood sarcoidosis in Denmark 1979-1994: incidence, clinical features and laboratory results at presentation in 48 children. Acta Paediatr 2004;93(1):30-6.

11. Rodriguez GE, Shin BC, Abernathy RS, Kendig ELJr. Serum angiotensin-converting enzyme activity in normal children and in those with sarcoidosis. J Pediatr 1981;99(1):68-72.

12. Baculard A, Blanc N, Boulé M, Fauroux B, et al. Pulmonary sarcoidosis in children: a follow-up study. Eur Respir 2001;17(4):628-35.

13. Judson MA. The treatment of pulmonary sarcoidosis. Respir Med 2012;106(10):1351-61.

14. Rosen Y. Pathology of sarcoidosis. Semin Respir Crit Care Med 2007;28(1):36-52.

15. Sekine T, Amano Y,Hidaka F, Takagi R, et al.Hepatosplenic and muscular sarcoidosis: characterization with $\mathrm{MR}$ imaging. Magn Reson Med Sci 2012;11(2):83-9. 